\title{
Los Municipios Chilenos y la Inversión Pública
}

\author{
Autor: \\ Cristian Loyola Díaz ${ }^{29}$ \\ Fecha de Recepción: 10/07/2012 \\ Fecha de Aceptación: 15/08/20I2
}

\section{Resumen}

Este artículo analiza la inversión pública que se realiza en Chile, poniendo el foco de atención en cuál es el rol que juegan los municipios en dicho ámbito. En este sentido, se realiza una revisión de los datos proporcionados por el Sistema Nacional de Inversiones (SNI) y el Banco Integrado de Proyectos (BIP), administrados por el Ministerio de Desarrollo Social y el Ministerio de Hacienda, para el período 2009 -20I I, con la finalidad de recabar cuál es el escenario en la actualidad. La principal conclusión es que los municipios en Chile tienen escaso poder en materia de inversión pública porque la formulación de proyectos que realizan es de baja calidad y tamaño; de esta manera, no obtienen una recomendación favorable para su financiamiento. A partir de esta premisa, la pregunta que emerge como desafío es acaso los gobiernos locales están dotados de competencias adecuadas para cumplir estas labores y si el Estado Central tiene los incentivos necesarios para generar nuevas competencias y permitir, de esta manera, que los municipios se transformen en actores importantes en el ciclo de la inversión pública.

Palabras claves: Inversión pública - Municipios - Capital humano - Gestión de proyectos de inversión

\section{Abstract}

This article analyzes public investment in Chile focusing on the role played by municipalities in this field.To do soit's made a review of the data provided by the National Investment System (NIS) and the Integrated Bank of Projects (BIP), administrated by the Ministry of Social Development and Ministry of Treasury for the period 2009 -20II with the purpose of presenting today's scenario. The main conclusion is that the Chilean municipalities have little power in public investment because projects they formulate and perform are of low quality and amount; thus, they do not get a favorable recommendation for funding. From this premise, the question that arises as a challenge is whether or not local governments are provided with appropriate skills to fulfill these tasks and if the Central Government has stimulies to build new competencies that could allow municipalities tobecome major players in the cycle of public investment.

Keywords: Public Investment - Municipalities - Human Capital - Management of Investment Projects 


\section{Resumo}

Este artigo analisa a inversão pública que realiza em Chile, colocando o foco da atenção no qual é o rol que jogam os municípios em este âmbito. Em este sentido, se realiza uma revisão dos dados proporcionados pelo Sistema Nacional de Inversões (SNI) y o Banco Integrado de Projetos (BIP), administrados pelo Ministério de Desenvolvimento Social e o Ministério da Fazenda , para o período 2009 -20II, com a finalidade solicitar qual é a situação na atualidade.A principal conclusão é que os municípios em Chile tem pouco poder em matéria de inversão pública porque a formulação de projetos que realizam é de baixa qualidade e tamanho; de esta maneira, não obtém uma recomendação favorável para seu financiamento. A partir de esta premissa, a pergunta que emerge como desafio é acaso os governos locais estão dotados de competências adequadas para cumprir estas funções se o Estado Central tem os incentivos necessários para gerar novas competências e permitir, de esta maneira, que os municípios se transformem em atores importantes no ciclo da inversão pública.

Palavras-Chave: Inversão pública - Municípios - Capital humano - Gestão de projetos de inversão

\section{Introducción}

El presente artículo tiene como objetivo analizar la inversión pública que se realiza en el país, poniendo el foco de atención en cuál es el rol que juegan los municipios en dicho ámbito. Con tal propósito, se realiza una revisión de los datos proporcionados por el Sistema Nacional de Inversiones (SNI) y el Banco Integrado de Proyectos (BIP), ambos administrados por el Ministerio de Desarrollo Social, para el periodo 2009 -20I I, con la finalidad de presentar cuál es el escenario de inversión pública en que se desenvuelven los municipios en la actualidad.

Indudablemente, la Inversión Pública y la participación de los municipios en ésta, es sólo una excusa para hablar de un nuevo foco de exclusión en la que operan los gobiernos locales. Porque la cantidad de proyectos ejecutados y el financiamiento involucrado por las municipalidades invita inmediatamente a revisar temas como recursos humanos, remuneraciones, especialización, entre otros. El objetivo de este documento es, entonces, diagnosticar, desde el punto de vista de la Inversión Pública, los síntomas del centralismo bajo el cual históricamente se ha configurado el país. En este sentido, más que responder preguntas, la intención del artículo es abrir preguntas al debate, especialmente en miras a las discusiones que actualmente se están desarrollando en función de la Reforma Municipal.

\section{Descripción del Sistema Nacional de Inversiones}

El Sistema Nacional de Inversiones (SNI, de ahora en adelante) corresponde a un cuerpo de normas técnicas, procesos y procedimientos configurados para establecer criterios de efectividad y eficiencia en la utilización de los recursos públicos que se destinan a inversión pública en Chile. La operación de este sistema es de responsabilidad del Ministerio de Desarrollo Social (ex Mideplan) y del Ministerio de Hacienda.

Para su operación, el SNI se compone de cuatro subsistemas, cada un asociado a una etapa del proceso de inversión, cuales son: 


\section{- Subsistema de Análisis Técnico Económico \\ - Subsistema de Formulación Presupuestaria \\ - Subsistema de Ejecución Presupuestaria \\ - Subsistema de Evaluación Ex Post}

Figura $\mathrm{N}^{\circ}$ I. Fases del proceso de inversión pública en Chile, regulado por el SNI

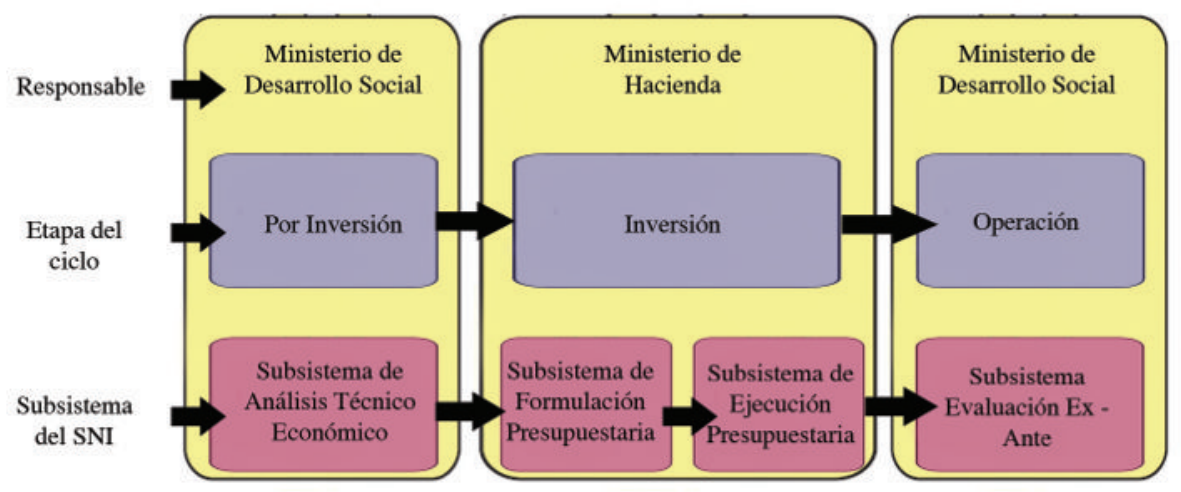

Fuente: Elaboración propia.

El subsistema Análisis Técnico Económico permite que las instituciones públicas dispongan de una cartera de iniciativas de inversión socialmente rentables y que, por tanto, estén en condiciones de recibir recursos públicos para su ejecución. La administración de este proceso recae íntegramente en el Ministerio de Desarrollo Social.

Por su parte, el subsistema Formulación Presupuestaria corresponde a la asignación de recursos financieros a los proyectos de interés sectorial, regional y de las empresas del Estado. Su función es homogeneizar, normar y coordinar la información relativa al proceso de asignación de fondos, así como también la aplicación del presupuesto aprobado para el sector público. Este subsistema es responsabilidad del Ministerio de Hacienda.

El subsistema Ejecución Presupuestaria corresponde a la regulación y supervisión de la ejecución del gasto público y su respectivo financiamiento, materializada a través de la Ley de Presupuestos del Sector Público, anual, y las instrucciones complementarias de la Dirección de Presupuestos del Ministerio de Hacienda (Dipres).

Finalmente, el subsistema de Evaluación Ex Post corresponde al análisis de los resultados logrados una vez que el proyecto entra en operación, para medir el grado de cumplimiento de los objetivos propuestos, retroalimentar y actualizar las metodologías, parámetros y supuestos del análisis técnico económico (evaluación ex ante), y así poder efectuar las correcciones tendientes a perfeccionar los procesos y los procedimientos de inversión vigentes. Este subsistema es también responsabilidad del Ministerio de Desarrollo Social, quien anualmente realiza una evaluación ex post simplificada de una muestra representativa de proyectos, programas y estudios básicos financiados por los gobiernos regionales. 


\section{II.Actores que Intervienen en el SNI}

La figura que sigue a continuación presenta un esquema funcional y territorial de los distintos actores que participan en el Sistema Nacional de Inversiones (SNI). Como se puede apreciar, funcionalmente los roles de los actores se han clasificado en Asesoría, Gobierno y Administración; y desde el punto de vista territorial, se han clasificado en nacional, regional y municipal.

Figura $N^{\circ} 2$. Esquema Actores y Funciones del Sistema Nacional de Inversiones

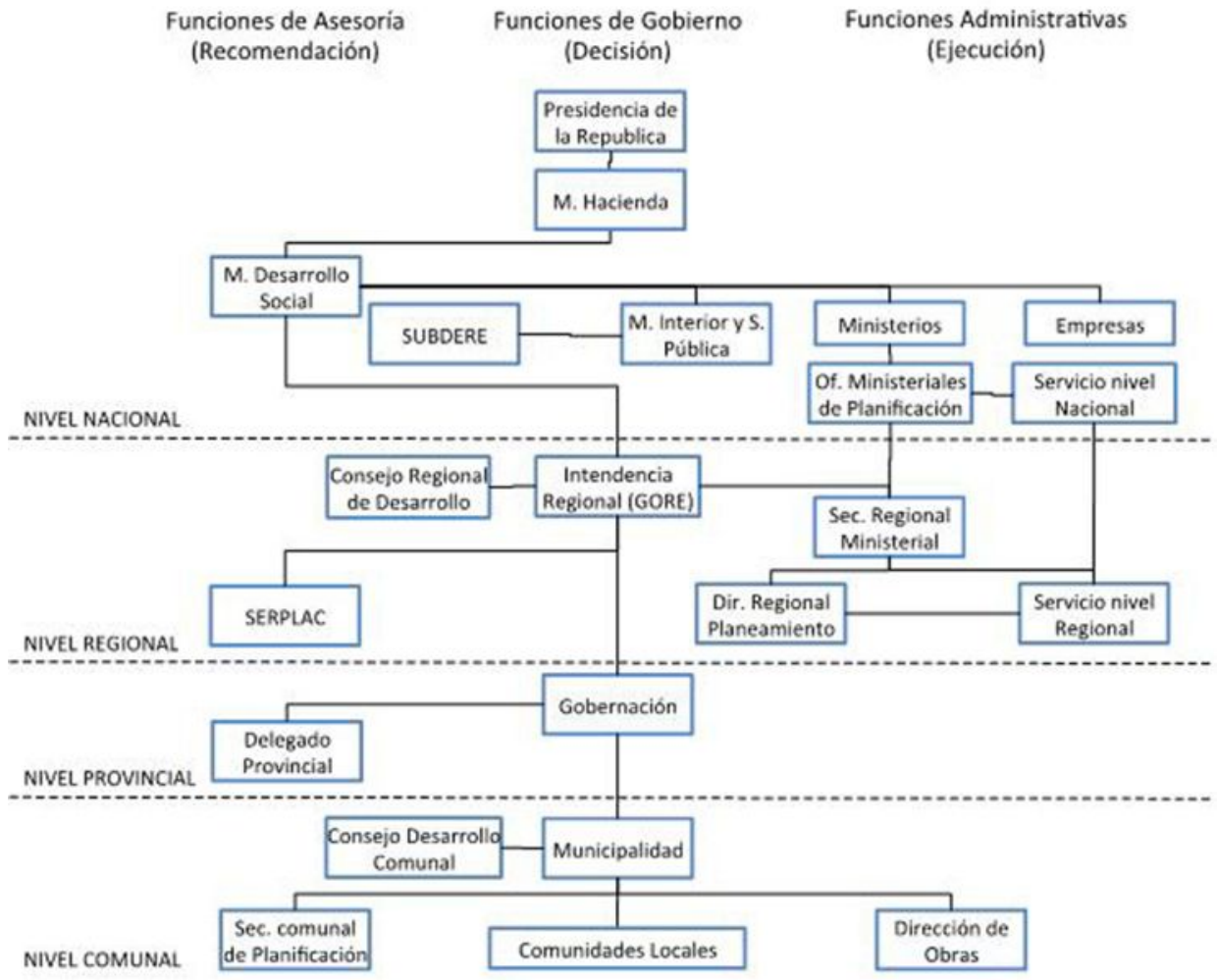

III.Antecedentes de la Inversión Pública

Considerando el marco descriptivo de la institucionalidad bajo la cual opera la inversión pública en Chile, es interesante observar la magnitud de la participación de los distintos actores descritos. Esto es posible de visualizar teniendo en consideración el Presupuesto anual en Inversión Pública y cuál es el porcentaje de gasto que cada uno de ellos ejecuta. En este sentido, se puede distinguir claramente que la inversión pública en su mayor proporción es definida por el gobierno central a través de sus distintos ministerios. Como se puede observar en el Gráfico $N^{\circ}$ I, la participación, o más bien formulación, en la inversión pública desde las municipalidades es bastante restringida. Desde la década de los noventa, ésta correspondía a poco más del 17\% del Presupuesto anual que el Estado dispone, bajando sistemáticamente hasta ubicarse en el 6,9\% para el 2007. 
Gráfico $N^{\circ}$ I. Participación porcentual de los actores en la toma de decisiones del Presupuesto 2007 de Inversión Pública

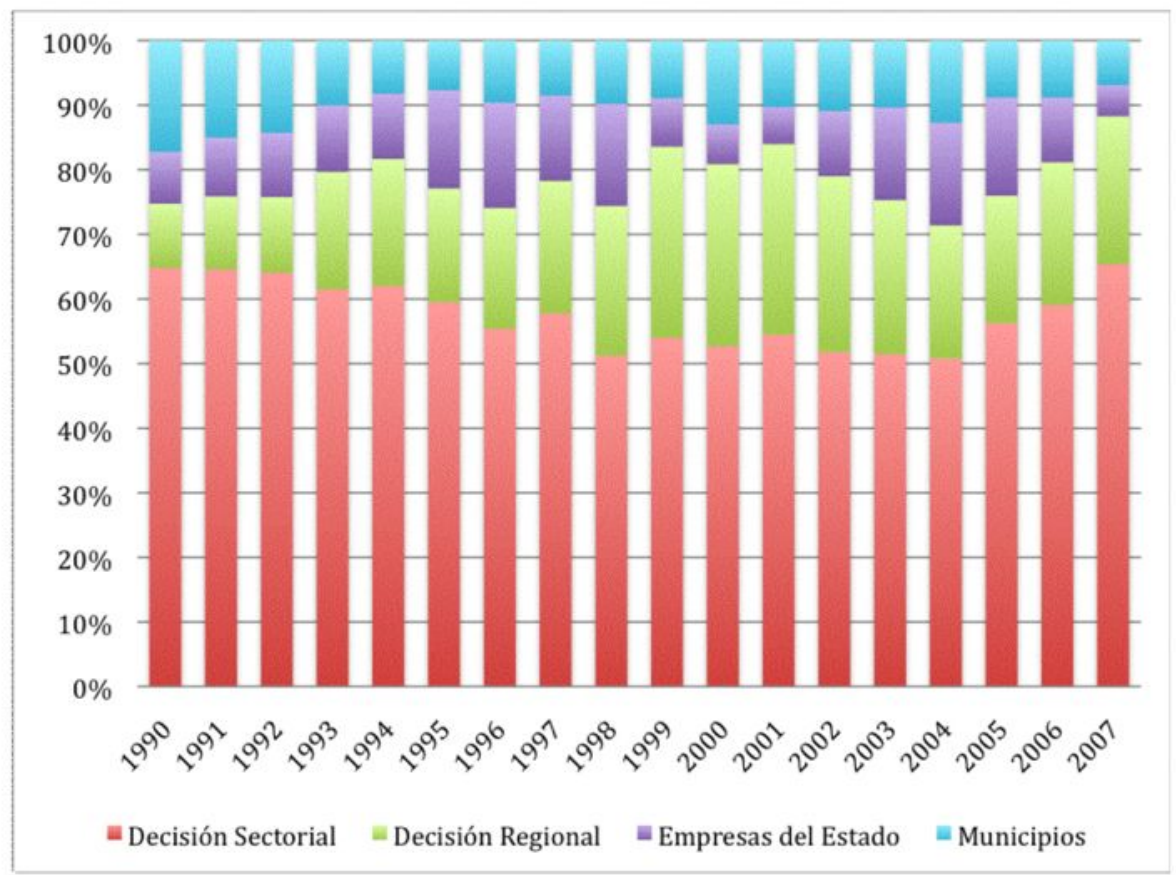

Fuente: Libertad y Desarrollo.

En este escenario, la pregunta central dice relación con el rol que juegan los Municipios del país en la contribución tanto de la generación como del análisis de las iniciativas de inversión. La primera hipótesis que surge es que, dado que las municipalidades no cuentan con capacidad para contribuir a la generación y evaluación de iniciativas de inversión pública, el principio de autonomíaque las caracteriza corresponde a un concepto más bien relativo y no absoluto. Pudiendo comprobar esta primera afirmación, la segunda hipótesis es que el foco del Estado no está puesto en generar e instalar competencias adecuadas para que los distintos municipios sean capaces de participar en los distintos procesos de inversión pública. De esta forma, el problema de estudio que se pretende instalar desde el análisis propuesto permite sustentar juicios en relación a la promoción de la política local desde el Estado.

IV.Análisis de las Cifras de Inversión Pública

Como se ha señalado al inicio del artículo, el horizonte temporal bajo el cual se analiza la Inversión Pública en Chile corresponde al intervalo de años 2009 - 201 I. Bajo dicho marco, lo primero que habría que señalar dice relación con la totalidad de iniciativas de inversión que obtienen una recomendación positiva (RS) para su ejecución; como se presenta en el Gráfico $N^{\circ}$ 2,si se toma el 2009 como año 0, los períodos posteriores muestran una baja en la cantidad de proyectos. Si para el año 0, vale decir 2009, el BIP 
registra 7.324 iniciativas de inversión (IDI's), para el año 201 I se muestra una disminución del $8 \%$, con un registro de 6.768 proyectos y programas en total.

Gráfico $N^{\circ}$ 2. Número de Proyectos con recomendación positiva (RS) por año, del Banco Integrado de Proyectos, 2009

2011

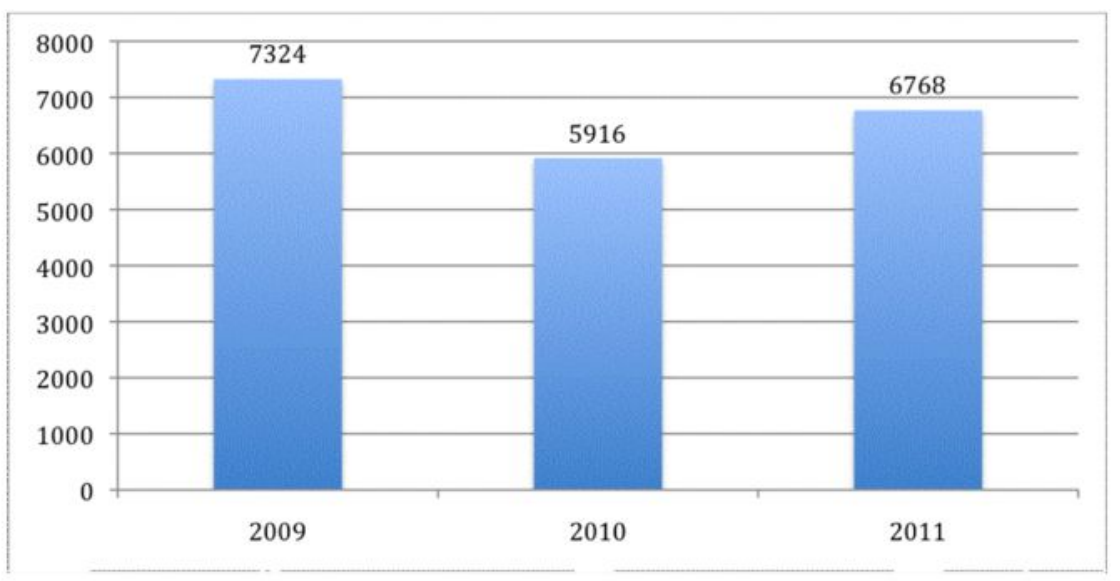

Fuente: Elaboración propia en base a información provista por el BIP.

Sin embargo, la cantidad de Iniciativas de Inversión (IDI's) ingresados por un actor al BIP no puede ser considerada una medida de evaluación absoluta de la Inversión Pública. Como se puede apreciar en el Gráfico $\mathrm{N}^{\circ} 3$, los montos destinados a la ejecución de programas y proyectos también sufre una baja de 2009 a 201 I, siendo la más notable la que se observa en 2010 .

Gráfico $N^{\circ}$ 3. Monto Presupuesto anual de la Nación, disponible para Inversión Pública, $2009-201 \mathrm{I}(\mathrm{M} \$)$

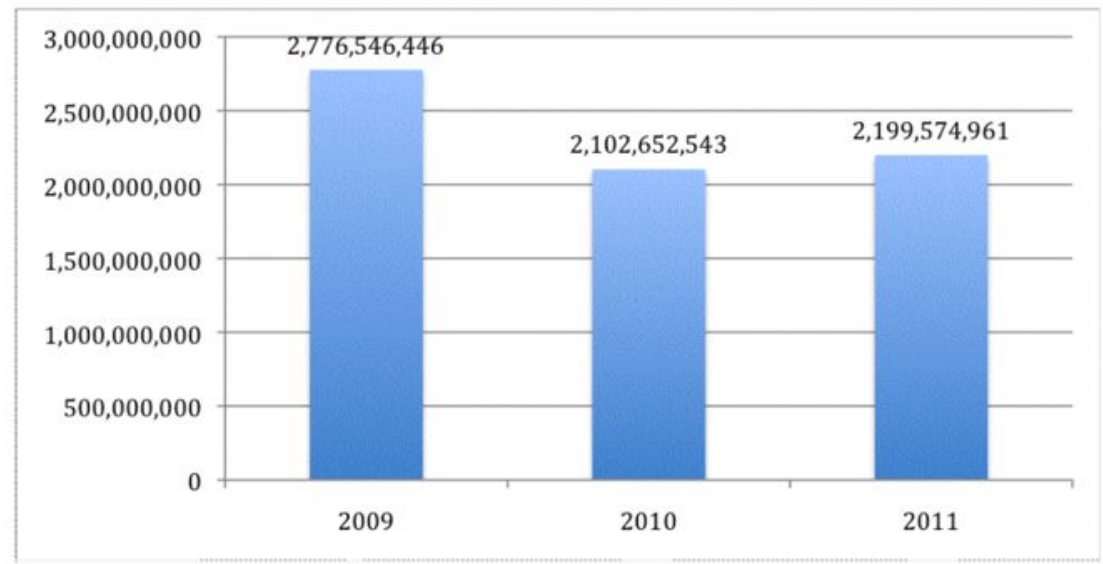

Fuente: Elaboración propia en base a información provista por el BIP. 
De acuerdo a la información entregada por el BIP, las fuentes que financiaron las iniciativas de inversión para el periodo de análisis son tres: Fondo Nacional de Desarrollo Regional (FNDR), Sectorial y Municipal. Al desglosar la cantidad de iniciativas de inversión con recomendación favorable para su ejecución, es posible comprobar que aquellas con financiamiento del FNDR corresponden a la mayoría de la cartera de proyectos. A su vez, resulta interesante constatar que las fuentes municipales fluctúan entre el $5 \%$ y $3 \%$ del total de iniciativas de inversión pública.Ver Gráfico $\mathrm{N}^{\mathrm{a}} 4$.

Gráfico $N^{\circ}$ 4. Porcentaje de iniciativas de inversión con recomendación favorable, por año y fuente de financiamiento,2009 - 20I I

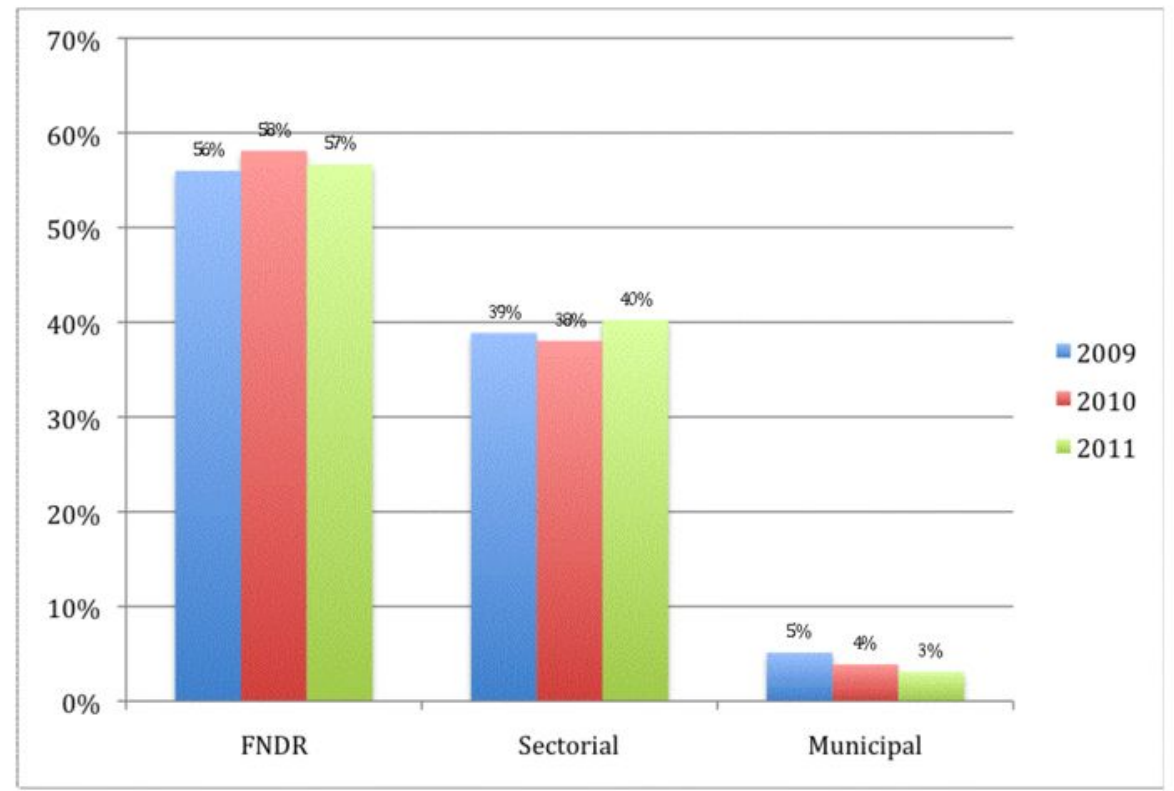

Fuente: Elaboración propia en base a información provista por el BIP.

El Gráfico $N^{\circ} 5$ muestrade mejor forma el lugar que ocupa el Municipio en la Inversión Pública. Se puede apreciar que desde el 2009 al año 201 I la inversión con financiamiento sectorial disminuye, pasando de un $69 \%$ a un $56,9 \%$. Esto tiene como principal correlato el crecimiento del FNDR, que para la totalidad de proyectos aprobados en el periodo de análisis, pasa de un 30,5\% de participación a un $42,5 \%$. No obstante, como se observa, el financiamiento municipal de la inversión pública no tiene grandes cambios. A su vez, es posible señalar que la participación de estos fondos es absolutamente marginal en relación a las otras dos fuentes descritas anteriormente. 
Gráfico $N^{\circ}$ 5. Porcentaje anual de Inversión Pública, según fuente de financiamiento, $2009-2011$

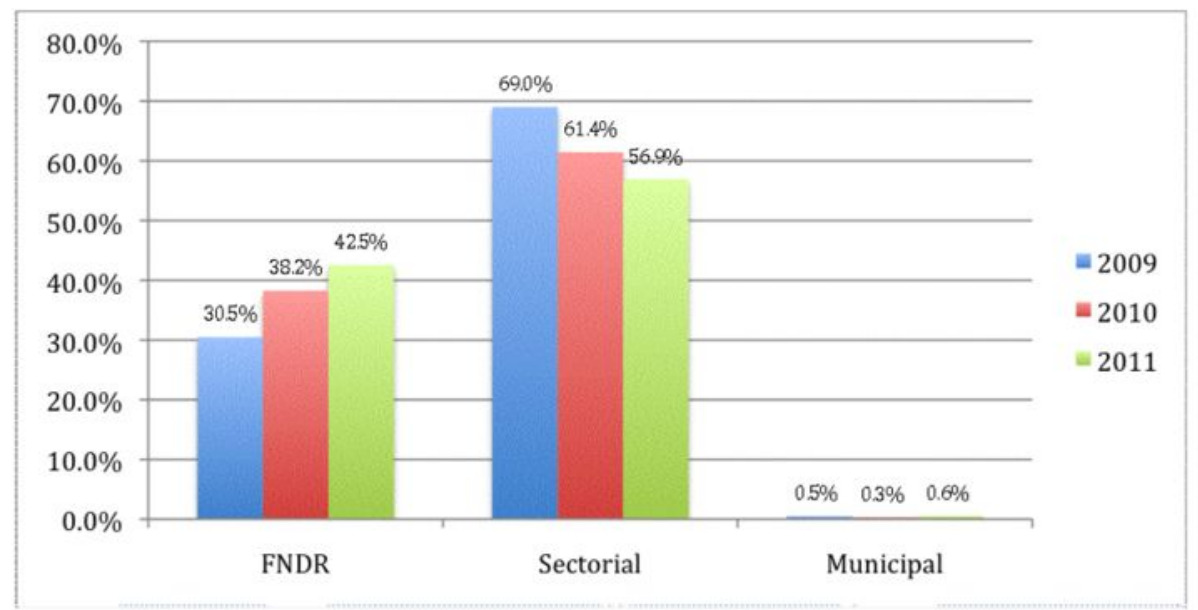

A nivel municipal, es posible señalar que existe un bajo número de comunas que lograron al menos una iniciativa de inversión con recomendación favorable en el SNI. Como se puede apreciar en el Gráfico N 6, el año 2009 se alcanza el máximo de participación de los municipios en la cartera de inversión del BIP: 183, es decir, un $53 \%$ de los municipios del país. Para los años siguientes, no obstante, esta presencia municipal en la inversión pública disminuye notablemente, alcanzando tan sólo un $32 \%$ de los municipios del país.

Gráfico $N^{\circ}$ 6. Número de municipalidades que logran al menos una iniciativa de inversión con recomendación favorable en el SNI, 2009 - $20 \mathrm{I} \mathrm{I}$

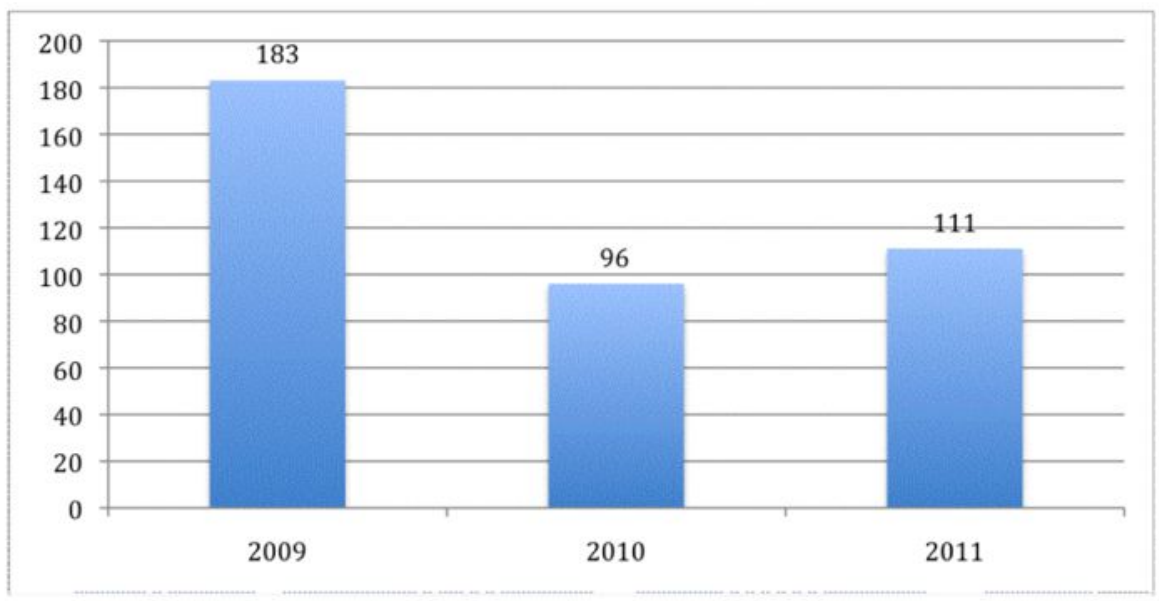

Fuente: Elaboración propia en base a información provista por el BIP.

Si se registra la distribución regional de los municipios participantes en la cartera de inversión públicadurante el periodo de evaluación 2009 - 20l I, se observa que tanto 
la Región Metropolitana como la Región del Biobío son por lejos las que concentran la mayoría de los programas o proyectos recomendados favorablemente: 80 y 79 , respectivamente. Por otro lado, las regiones con la menor participación corresponden a Tarapacá y Magallanes, con 6 y 7 respectivamente.

Gráfico $\mathrm{N}^{\circ}$ 7. Número de programas o proyectos recomendados favorablemente por región en el SNI, período 2009 - 2011

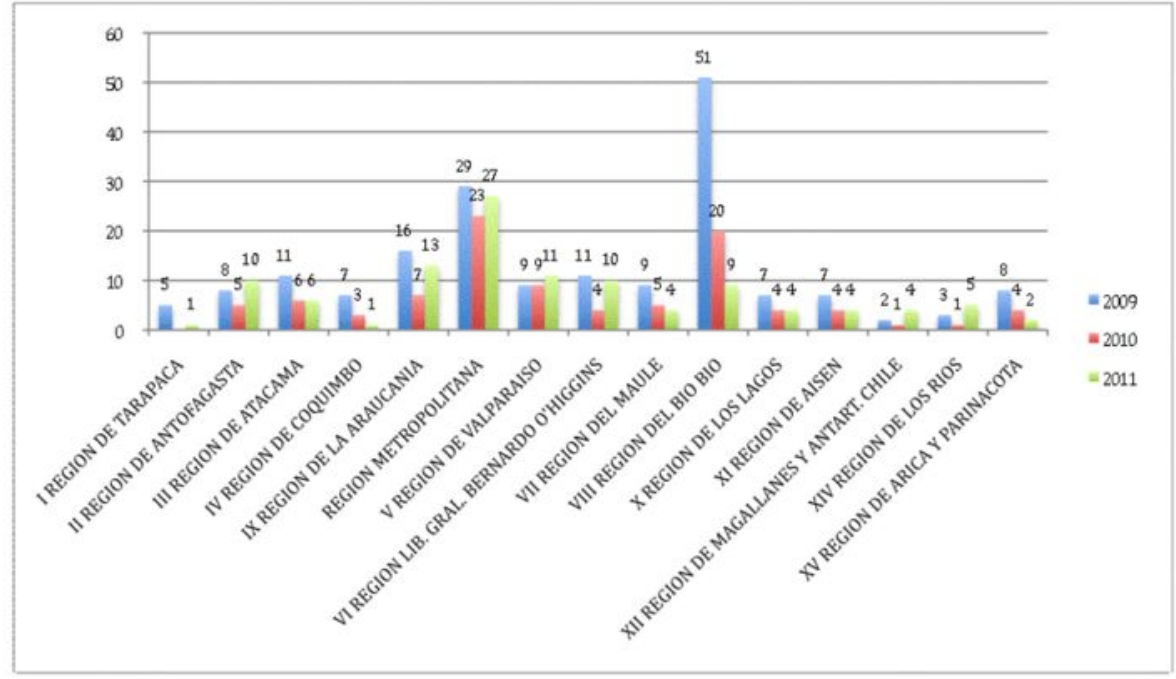

Fuente: Elaboración propia en base a información provista por el BIP.

Desde el punto de vista financiero, se puede señalar que la Región de Antofagasta es la que mayor financiamiento ha obtenido por iniciativas de inversión recomendadas favorablemente, sumando en el periodo 2009 - 201 I casi $M \$ 8.000 .000$. Esto puede explicarse por los resultados del año $201 \mathrm{I}$, en donde notoriamente los municipios de la Región pudieron aprobar proyectos de gran envergadura, como por ejemplo, la normalización del Estadio Regional, por un monto de $M \$ 2.000 .000$. Distinto es el caso de la Región de Atacama, que es la que menos recursos pudo obtener, alcanzando poco más de $M \$ 380.000$. 
Gráfico $N^{\circ}$ 8. Monto de financiamiento comprometido en iniciativas de inversión recomendadas favorablemente en el SNI, por región, en M\$, 2009-20I I

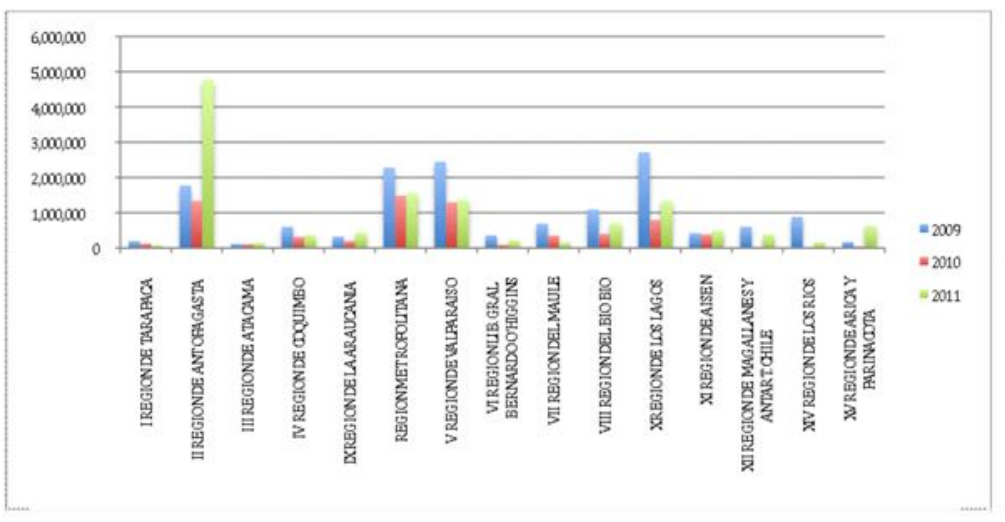

Fuente: Elaboración propia en base a información provista por el BIP.

Una visión sobre el costo de los proyectos también es interesante de revisar. Un primer punto que llama la atención dice relación con que los proyectos de menor financiamiento, vale decir, aquellos con un costo de hasta $M \$ 100.000$, que son los que dominan la cartera de Iniciativas de Inversión municipal, representando el 93\%. A su vez, el promedio de costo en esta categoría es bastante bajo y corresponde a $M \$ 19.425$. En este sentido, se puede evaluar que los proyectos de tipo municipal que forman parte de la Inversión Pública corresponden a los de menor envergadura y costo. Así mismo, la totalidad de proyectos presentados por los municipios tienen un costo promedio de $M \$ 42.343$, muy menor el promedio de $M \$ 227.349$ de los FNDR y los $M \$ 570.170$ correspondiente a los sectoriales.

Tabla $\mathrm{N}^{\circ}$ I. Cantidad de proyectos municipales con recomendación favorable en el SNI, por rango de monto de financiamiento, porcentaje y promedio 2009 - 201 I

\begin{tabular}{|l|l|l|l}
\hline Rango en M\$ & $\begin{array}{l}\mathrm{N}^{\circ} \text { de proy ectos } \\
\text { recomendados }\end{array}$ & Porcentaje & $\begin{array}{l}\text { Monto } \\
\text { promedio }\end{array}$ \\
\hline $0-100.000$ & 762 & $93,38 \%$ & $\$ 19.425$ \\
\hline $100.001-200.000$ & 29 & $3,55 \%$ & $\$ 147.158$ \\
\hline $200.001-300.000$ & 3 & $0,37 \%$ & $\$ 250.110$ \\
\hline $300.001-400.000$ & 6 & $0,74 \%$ & $\$ 355.728$ \\
\hline $400.001-500.000$ & 4 & $0,49 \%$ & $\$ 469.245$ \\
\hline $500.001-600.000$ & 5 & $0,61 \%$ & $\$ 537.067$ \\
\hline $600.001-700.000$ & 1 & $0,12 \%$ & $\$ 691.180$ \\
\hline $700.001-800.000$ & 2 & $0,25 \%$ & $\$ 769.965$ \\
\hline $800.001-900.000$ & 0 & $0,00 \%$ & $\$ 0$ \\
\hline $900.001-1.000 .000$ & 1 & $0,12 \%$ & $\$ 910.001$ \\
\hline 1.000 .001 y más & 3 & $0,37 \%$ & $\$ 1.631 .347$ \\
\hline Total & 816 & $100,00 \%$ & $\$ 42.343$ \\
\hline
\end{tabular}

Fuente: Elaboración propia en base a información provista por el BIP. 


\section{Causas}

Los últimos años de desarrollo del país han sido exitosos en materia de crecimiento económico para Chile. Sin embargo, los frutos de este proceso no han sido acompañados de logros en materia de descentralización, tal como se ha presentado en los datos anteriores. Específicamente, para el contexto del presente estudio sobre la Inversión Pública, los municipios siguen siendo un actor excluido.

Tal como señala Contreras (2009), los municipios - en el contexto de la Inversión Pública - presentan carencias y limitaciones que no les permiten planificar su actuar en el mediano y largo plazo. En este sentido, se identifican dos grandes problemas que afectan directamente a la baja incidencia de los gobiernos locales en dicha inversión.

En primer término, existiría una baja pertinencia de iniciativas de inversión respecto de las necesidades u oportunidades de los espacios locales. En términos formales, las necesidades que demanda la ciudadanía respecto de sus municipios deben ser recogidas a través del Plan de Desarrollo Comunal (Pladeco). Sin embargo, según la Encuesta Nacional de Opinión Pública del ICHEM - 20I0, el 95,\%señala que no conoce el Pladeco de su comuna ${ }^{30}$. Más aún, según la misma encuesta, el $99 \%$ de la población indica que no ha participado en la elaboración de dicho Plan comunal.

En segundo lugar, los municipios del país que presentan Iniciativas de Inversión al SNI para su evaluación requieren de gran cantidad de tiempo para obtener una recomendación favorable. Como indica Contreras (2009), en promedio un municipio demora 174 días en obtener la recomendación favorable.A su vez, el $50 \%$ de los municipios demora entre 157 y 596 días. En otras palabras, estos datos hablan de una muy baja eficiencia en la presentación y evaluación ex ante de programas y proyectos de inversión.

Ahora bien, la pregunta que debiese ser respondida es ¿Por qué los municipios, especialmente, presentan estos problemas en el contexto de la Inversión Pública? En este sentido, uno de los principales factores que contribuye a fijar esta situación dice relación con la dotación de Recursos Humanos de las unidades municipales encargadas de formular programas y proyectos de inversión.

Según datos de la Subsecretaría de Desarrollo Regional (Subdere), de 2008, citados por Contreras (2009), las bajas remuneraciones contribuyen a este escenario: un 62,4\% de quienes trabajan en las áreas de planificación de los municipios tienen ingresos hasta $\$ 336.447$. El porcentaje aumenta al $95 \%$ si la cota máxima de ingresos es aumentada a $\$ 8 \mid$ 8.823. Esta situación es relevante, dado que genera un efecto en cadena: bajos sueldos implican una alta rotación y, a su vez, un bajo nivel de especialización.

30.- El dato no debe llamar a asombro, dada la lejanía de la ciudadanía con sus gobiernos locales. Por ejemplo, si se toma como fuente de información la misma Encuesta Nacional de Opinión Pública del ICHEM - 20I0, el $89 \%$ de la población no conoce el Plan Regulador, el $91 \%$ no sabe acerca del Presupuesto Municipal y el $94 \%$ no tiene información sobre el Plan Anual de Educación Municipal (Padem). 
Figura $\mathrm{N}^{\circ}$. Esquema de comportamiento dotación de Recursos Humanos en áreas de planificación municipal

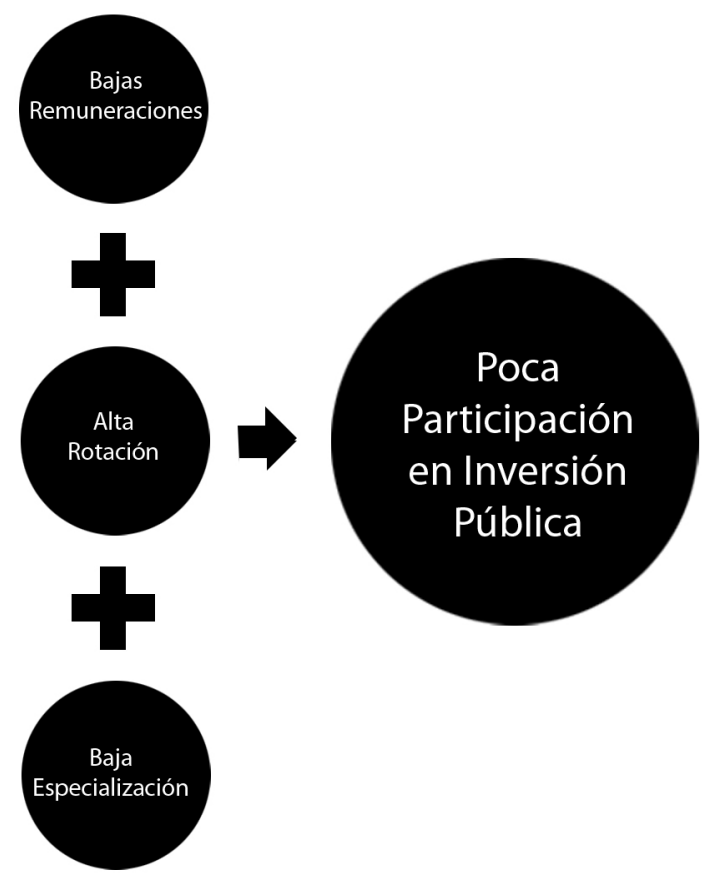

Fuente: Elaboración propia.

\section{Conclusiones}

Los datos presentados hacen necesaria la pregunta sobre cuál es el lugar y la importancia que tienen los municipios en la elaboración y decisión de la inversión pública en Chile. Como se ha podido desprender de la descripción de los organismos que intervienen en el proceso de inversión, el ciclo tiene una característica centralista muy marcada. No obstante, se reconocen esfuerzos de descentralización de la inversión pública, especialmente al analizar la evolución de la participación del FNDR en el total del presupuesto que se gasta en programas y proyectos de inversión. Sin embargo y tal como se ha constatado, este proceso corresponde a una descentralización que alcanza sólo hasta el nivel regional.

Como se ha evidenciado a través de la información proporcionada por los organismos públicos respecto del comportamiento de la Inversión Pública en Chile durante los últimos años, el municipio parece no estar presente en el foco de la discusión sobre la creación de programas y proyectos donde se invierten recursos públicos. La cantidad marginal de iniciativas con recomendación favorable, y los montos en dinero involucrados, así lo demuestran.

A su vez, como se ha descrito anteriormente, la recomendación favorable de las 
iniciativas de inversión en el SNlcumplen un largo y complejo ciclo, comenzando en su formulación y terminando en la evaluación ex post. De esa manera, uno de los factores que pudiese estar influyendo en la baja participación municipal dice relación con la falta de capital humano adecuado para realizar las tareas vinculadas al ciclo de un proyecto. Varios estudios confirman este ámbito como uno de los más críticos, como lo sostienen Arriagada $^{31}$ y Bernstein e Inostroza ${ }^{32}$. Esto se debe a varias razones, entre las cuales coinciden bajas remuneraciones y una alta rotación en el cuerpo profesional de los municipios.

Finalmente, el enfoque municipal acerca de la Inversión Pública debería cambiar. Además de ser modificados aspectos del funcionamiento mismo de los gobiernos locales para la formulación de programas y proyectos, es necesario que las Iniciativas de Inversión aspiren a tener un mayor impacto. Desde este punto de vista, cada uno de los Pladeco debe estar vinculado con esta tarea, porque es precisamente el instrumento pensado para detectar cuáles son las necesidades de la población en las comunas. Un cambio de este tipo, por ejemplo, permitiría la realización de inversiones con mucho mayor impacto que pavimentaciones de calles, las cuales alcanzan hoy en día al $50 \%$ de las Iniciativas de Inversión municipales recomendadas favorablemente.

Las preguntas que emergen ante este escenario son dos. En primer lugar, ¿El Estado considera importante que los municipios del país participen más y mejor en la Inversión Pública? Y, segundo, ¿Qué pueden hacer los municipios para mejorar su participación en la Inversión Pública?

Bibliografía

Contreras, E.,Barros, A.,González, N. \& Fuenzalida, J. (2009). Gestión del Conocimiento y Transparencia: Desafíos para la Inversión Pública a Nivel Local. Centro de Sistemas Públicos, Universidad de Chile.

Horst, B. (2009). Fuentes de Financiamiento para Gobiernos Subnacionales y descentralización Fiscal. Consorcio para la Reforma del Estado. Santiago de Chile.

Instituto Chileno de Estudios Municipales (ICHEM). (2010). Encuesta Nacional de Opinión Pública - 2010. Universidad Autónoma de Chile. Santiago de Chile.

Ministerio de Planificación. (20II). Definiciones estratégicas 20II. Dirección de Presupuestos, Ministerio de Hacienda de Chile.

3I.- Ricardo Arriagada. “Diseño de un Sistema de Medición de Desempeño para Evaluar la Gestión Municipal: una propuesta Metodológica”. CEPAL. 2002.

32.- Felipe Bernstein y José Inostroza. "Modernización municipal y un sistemade evaluación de su gestión.Propuesta de una arquitectura”. En: Un Mejor Estado Para Chile. 2010. 


\section{Páginas web}

Banco Integrado de Proyectos (BIP). http://bip.ministeriodesarrollosocial.gob.cl/biptrabajo/index.html

Instituto Chileno de Estudios Municipales.www.ichem.cl

Sistema Nacional de Inversiones (SNI). http://sni.ministeriodesarrollosocial.gob.cl/ 